\title{
Minimum-Link Watchman Tours
}

\author{
Esther M. Arkin* Joseph S. B. Mitchell ${ }^{\dagger} \quad$ Christine D. Piatko
}

\begin{abstract}
We consider the problem of computing a watchman route in a polygon with holes. We show that the problem of finding a minimum-link watchman route is NP-complete, even if the holes are all convex. The proof is based on showing that the related problem of finding a minimum-link tour on a set of points in the plane is NP-complete. We provide a provably good approximation algorithm that achieves an approximation factor of $O(\log n)$.
\end{abstract}

Keywords: link distance, watchman route, polygons, NP-complete, approximation algorithms, computational geometry

\section{Introduction}

The problem of finding a short watchman route according to the Euclidean metric has been extensively studied over the last ten years; see $[3,4,5,11,12,13]$. In the case of guarding a polygonal domain (with "holes"), the Euclidean version of the watchman route problem is NP-hard (from Euclidean TSP) [4], and there is an $O(\log n)$-approximation algorithm for a rectilinear version with restricted visibility [9]. In the case of guarding a simple polygon (no holes), Chin and Ntafos [5] gave an algorithm claimed to have time complexity $O\left(n^{4}\right)$ for the "fixed" watchman route through a specified starting point; their algorithm, as well as some of its successors, were later found to be flawed ([8]), and new methods were proposed (some of which were again flawed). Eventually, Tan, Hirata, and Inagaki re-established the $O\left(n^{4}\right)$ time bound on the fixed watchman route problem, using a new dynamic programming algorithm. Most recently, Tan [12] has given an $O\left(n^{5}\right)$ algorithm for the "floating" watchman route problem, in which no starting point is specified, building on the earlier work of Carlsson, Jonsson, and Nilsson [3], who gave the first techniques to handle the floating case.

Here, we investigate the watchman route problem in polygonal domains, with the objective to minimize the number of links in the (polygonal) route, rather than its Euclidean length. More specifically, given a polygon $P$ with holes (obstacles), we consider the problem of finding a minimum-link watchman route; i.e., we want to compute a minimum-link path within $P$ such that every point of $P$ is visible from at least one point along the route. We show that the problem of finding a minimumlink watchman route is NP-complete, and give a polynomial-time approximation algorithm with approximation factor $O(\log n)$ (where $n$ is the number of vertices in the polygonal scene).

*estie@ams.sunysb.edu; Department of Applied Mathematics and Statistics, Stony Brook University, Stony Brook, NY 11794-3600, USA. Partially supported by grants from HRL Laboratories (DARPA subcontract), the National Science Foundation (CCR-9732220,CCR-0098172), and Sandia National Labs.

†jsbm@ams.sunysb.edu; Department of Applied Mathematics and Statistics, Stony Brook University, Stony Brook, NY 11794-3600, USA. Partially supported by grants from the Binational Science Foundation (2000160), HRL Laboratories (DARPA subcontract), NASA Ames Research (NAG2-1325), the National Science Foundation (CCR9732220,CCR-0098172), Northrop-Grumman, and Sun Microsystems.

${ }^{\ddagger}$ Christine.Piatko@jhuapl.edu; JHU/APL, Johns Hopkins Road, Laurel, MD 20723-6099. 
Alsuwaiyel and Lee $[1,2]$ show that if $P$ is a simple polygon, then the minimum-link watchman route problem is NP-complete, and they give a constant-factor approximation algorithm. Their approximation algorithm does not at all apply to the case of holes (obstacles). Their hardness results would apply to the case of holes, if we allow the outer boundary of $P$ to be complex (so that their gadgets can be constructed). However, here we allow $P$ to be a polygon with a very simple outer boundary (it may have no outer boundary at all); for example, we allow $P$ to be a rectangle with convex holes. Even in this case, we are able to prove that the minimum-link watchman route problem is NP-complete.

\section{Hardness Proof}

We begin by showing that the problem of covering a set of points by a minimum-link tour is NP-hard, and then reduce it to a minimum-link watchman route in a polygon with holes.

Lemma 1 Given a set of points in the plane, it is NP-complete to determine whether they can be covered by a tour of $k$ links.

Proof. We give a reduction from the known NP-complete problem of determining whether a set of points in the plane can be covered by $r$ lines. The Point Covering BY Lines problem has been shown to be NP-complete by Megiddo and Tamir [10]. The idea of the reduction is to add additional points to the point set in such a way that covering the original points by lines will be equivalent to covering the enlarged point set by a tour.

Given an instance of the Point Covering By Lines problem, we construct an instance of the Point Covering BY A Tour problem, as follows: Set $k=3 r$. Build the line arrangement of the point set. Clearly, we can restrict all covering lines to be lines of this arrangement. Using an affine transformation (if needed), transform the arrangement so that we can find an infinite double wedge with the property that each of the arrangement lines has two infinite rays, one in each part of the double wedge. (Thus, only a finite line segment of each line lies outside of the double wedge.) We refer to one of the wedge parts as the right wedge and the other as the left wedge. Next, we build a series of $r$ infinite "V"s that are parallel to each other, each ray of the "V" crosses both the right and left wedges the double wedge, and they lie completely outside of the convex hul of all crossing points in the arrangement. The apices of the "V"s all lie on one side of the double wedge (say above the double wedge). The rays of the "V" are specified by $k+1$ points on each ray, all on the same side of the double wedge as the apices. See Figure 1 for $r=2$ and 4 original points.

We now show that the original point set can be covered by $r$ lines if and only if the enlarged point set can be covered by a tour of $k=3 r$ links. Given a tour of $3 r$ links covering the enlarged point set, it is easy to see that at least $2 r$ of the links are used to cover the new points. Removing these links yields a covering of the original point set by $r$ or fewer line segments.

Conversely, given a covering of the original point set by $r$ lines, we connect them into a covering tour that also covers the added points. Number the lines arbitrarily 1 to $r$. For $i=1, \ldots, r$ connect line $i$ to line $i+1(\bmod r)$ by one of the infinite "V"s. This requires deleting an infinite part of the lines and rays, but those parts deleted do not cover any points of the of the enlarged point set, by our construction. Since for every original line in the covering, we now have three line segments, we get the desired tour.

Theorem 1 Given a polyon $P$ with holes, it is NP-complete to determine whether there exists a watchman tour of $k$ links. In fact, even if $P$ is a convex polygon with convex holes, the problem is NP-complete. 


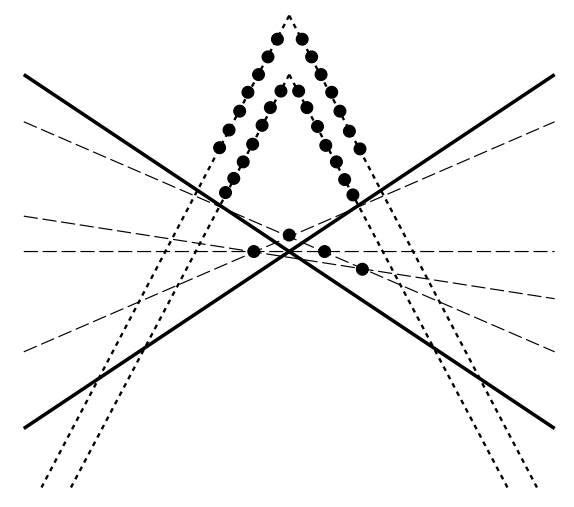

Figure 1: Constructing the "V's".

Proof. We use a reduction from the Point Covering By A Tour, shown to be NP-complete in the lemma above. Given an instance of the Point Covering BY A Tour problem, we enclose the point set in a large rectangle. Specifically, we build the arrangement of all lines passing through pairs of points in the point set, and make sure the rectangle includes all intersection points of the lines in its interior. It is easy to see that a min-link tour cover will consist of segments that are part of the line arrangement. Our idea is simple: Build small obstacles around each point in the set, such that a watchman route that sees a small circle around the point must visit the point (or some point very close to it). Furthermore, these obstacles will not block any line segment of the arrangement. To build the desired obstacles, draw two circles around each point, of radii $\epsilon$ and $2 \epsilon$. These circles will be cut by at least two lines of the arrangement. Each obstacle is a trapezoid with its two non-parallel sides lying on consecutive (around the point) lines of the arrangement between the two circles. See Figure 2. It is now easy to see that the points within the circle of radius $\epsilon$ (which are not interior to an obstacle) will not be visible to a watchman, unless the route visits this circle. For points on the boundary of the convex hull of the point set, we include only those obstacles interior to this convex hull, thus ensuring that once all points are visited, the remaining interior of the rectangle (namely the rectangle minus the convex hull of the point set) is visible.

We have thus argued that a watchman route must "visit" all points in the point set. By visit we mean the tour contains points with the $\epsilon$ ball around the point. Such a tour can be perturbed to visit the point itself, without increasing the number of links. It can be shown that a covering tour yields a watchman tour that sees the entire interior of the rectangle.

\section{An Approximation Algorithm}

Theorem 2 There exists a polynomial-time algorithm to compute an $O(\log n)$-approximation for the minimum-link watchman problem in a polygon $P$ (with holes) having a total of $n$ sides.

Proof. First, we construct the visibility graph for $P$; this can be done in time $O(E+n \log n)$, where $E$ is the number of edges in the visibility graph [7]. Next, we construct the arrangement of 


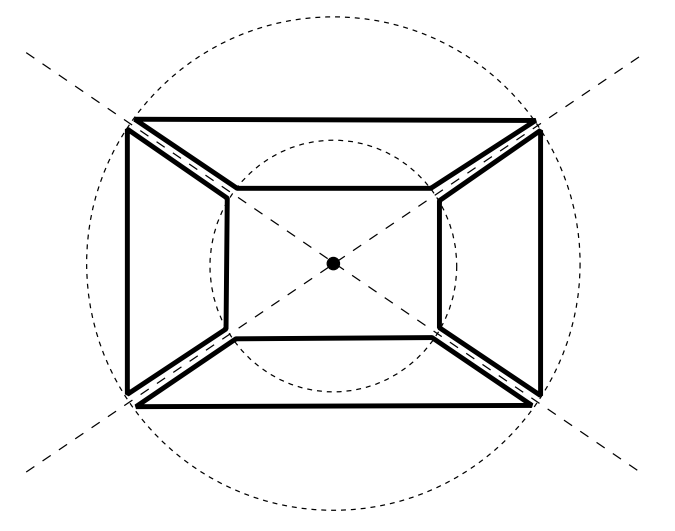

Figure 2: Building trapezoidal obstacles around intersection points of the arrangement

all extended visibility graph edges (i.e., visibility graph edges that have been extended to maximal length within $P$ ); this can be done in time $O\left(E^{2}\right)$.

Next, interior to each cell of this arrangement we place a viewpoint. For each viewpoint, we compute the visibility polygon within $P$. The boundary of each such visibility polygon consists of edges of $P$ and of "window" segments. Let $S$ denote the set of all window segments.

Consider any line segment $\sigma \subset P$, and let $S_{\sigma}$ denote the set of windows stabbed by $\sigma$. We extend $\sigma$ until its endpoints reach the boundary of $P$; this can only increase the set of windows stabbed by $\sigma$. Next, we rotate $\sigma$, while allowing its length to increase/decrease in order to keep its endpoints on the boundary of $P$, until it is "pinned" by some pair of window endpoints. The resulting segment is said to be a canonical chord of $P$. There are at most $O\left(n^{2} E^{4}\right)$ such canonical chords.

Let $\sigma$ be a canonical chord, and let $e_{1}$ and $e_{2}$ be the two (distinct) edges on the boundary of $P$ that contain the endpoints of $\sigma$. Then we call the set $\sigma \cup e_{1} \cup e_{2}$ the $H$-structure associated with $\sigma$, and we denote it by $H_{\sigma}$.

Now we consider an abstract set cover problem in which the "elements" are the visibility polygons corresponding to viewpoints and the "sets" are the H-structures corresponding to the canonical chords; an "element" belongs to a "set" iff the corresponding H-structure intersects at least one window of the visibility polygon associated with the corresponding viewpoint.

The greedy set cover heuristic is known to produce a collection of sets that cover all points, such that the number of sets is at most $O(\log n)$ times that of an optimal cover [6]. We show that any set of $k$ covering H-structures can be converted into a watchman tour having at most $O(k)$ links. First, we connect the $\mathrm{H}$-structures into a tree using no more than $2(k-1)$ additional (not necessarily canonical) chords. This is easy to do, since at any intermediate step, there must be two connected components of the forest obtained so far, with each of the two components seeing a common point within $P$. (Since the forest "sees" all of $P$, the link distance between any pair of H-structures in a covering of $P$ must be at most 2.) By doubling the resulting tree, we obtain a tour, $T_{H}$, with at most $8 k+6(k-1)=14 k-6=O(k)$ links. (The first term counts the double traversal of each H-structure and the second term accounts for the double traversal of the connections and the partitioning of some of the original chords, which need to be doubled.)

Let $p^{*}$ (resp., $\left.c^{*}\right)$ be the minimum number of chords (resp., links in a path) that illuminate all of $P$. Clearly $c^{*} \leq p^{*}$. Note that any covering by chords can be converted to a covering by the 
same number of canonical chords. Thus $c^{*}$ is also the minimum number of canonical chords that illuminate $P$. Let $c_{H}$ be the number of canonical chords obtained using the set cover heuristic, and let $p_{H}$ be the number of links in the tour $T_{H}$ obtained from the $\mathrm{H}$-structures corresponding to these $c_{H}$ chords. Finally, we have:

$$
p_{H} \leq 14 c_{H} \leq\left(14 \log n c^{*}\right) \leq(14 \log n) p^{*} .
$$

To complete the proof, we show that the path obtained by our heuristic "sees" the entire interior of the polygon $P$.

Lemma 2 Let $C$ be a set of canonical chords that illuminate all viewpoints (i.e., intersect the visibility polygon of each viewpoint of $P$ ). Then, the set of all $H$-structures that correspond to $C$ illuminates all of $P$.

Proof. Assume to the contrary that there is a point, $p$, that is not illuminated by the set of H-structures. Now, $p$ lies in some cell, $C$, of the arrangement of extended visibility graph edges. Using a line segment, $s$, connect the viewpoint for cell $C$ to the H-structure that sees it. "Slide"

one endpoint of $s$ from the viewpoint to the unseen point $p$, while keeping the other endpoint of $s$ on the H-structure, while keeping $s$ within $P$. This may rotate and translate the connecting segment $s$. We claim that the sliding described cannot "get stuck" since this would mean that the connecting segment is pinned by two vertices, and hence is part of an extension of a visibility graph edge. This in turn implies that the viewpoint and the unseen point are in different cells, contrary to our construction. Finally, the resulting segment connecting the unseen point $p$ to the H-structure is a witness to the fact that $p$ is seen.

\section{References}

[1] M. H. Alsuwaiyel and D. T. Lee. Minimal link visibility paths inside a simple polygon. Comput. Geom. Theory Appl., 3(1):1-25, 1993.

[2] M. H. Alsuwaiyel and D. T. Lee. Finding an approximate minimum-link visibility path inside a simple polygon. Inform. Process. Lett., 55(2):75-79, 1995.

[3] S. Carlsson, H. Jonsson, and B. J. Nilsson. Finding the shortest watchman route in a simple polygon. Discrete Comput. Geom., 22(3):377-402, 1999.

[4] W. Chin and S. Ntafos. Optimum watchman routes. Inform. Process. Lett., 28:39-44, 1988.

[5] W.-P. Chin and S. Ntafos. Shortest watchman routes in simple polygons. Discrete Comput. Geom., 6(1):9-31, 1991.

[6] T. H. Cormen, C. E. Leiserson, and R. L. Rivest. Introduction to Algorithms. MIT Press, Cambridge, MA, 1990.

[7] S. K. Ghosh and D. M. Mount. An output-sensitive algorithm for computing visibility graphs. SIAM J. Comput., 20:888-910, 1991. 
[8] M. Hammar and B. J. Nilsson. Concerning the time bounds of existing shortest watchman route algorithms. In Proc. 11th International Symposium on Fundamentals of Computation Theory, volume 1279 of Lecture Notes Comput. Sci., pages 210-221. Springer-Verlag, Sept. 1997.

[9] C. Mata and J. S. B. Mitchell. Approximation algorithms for geometric tour and network design problems. In Proc. 11th Annu. ACM Sympos. Comput. Geom., pages 360-369, 1995.

[10] N. Megiddo and A. Tamir. On the complexity of locating linear facilities in the plane. Oper. Res. Lett., 1:194-197, 1982.

[11] B. J. Nilsson. Guarding Art Galleries - Methods for Mobile Guards. PhD thesis, Lund University, 1995.

[12] X. Tan. Fast computation of shortest watchman routes in simple polygons. Inf. Proc. Lett., $77: 27-33,2001$.

[13] X. Tan, T. Hirata, and Y. Inagaki. Corrigendum to "An incremental algorithm for constructing shortest watchman routes". Internat. J. Comput. Geom. Appl., 9(3):319-323, 1999. 